PREHOSPITAL CARE

\title{
Surviving out of hospital cardiac arrest at home: a postcode lottery?
}

\author{
R M Lyon, S M Cobbe, J M Bradley, N R Grubb
}

Emerg Med J 2004;21:619-624. doi: 10.1136/emj.2003.010363

See end of article for authors' affiliations ......................

Correspondence to: Dr R M Lyon, c/o Dr N Grubb, Department of Cardiology, Royal Infirmary of Edinburgh, 51 Little France Crescent, Old Dalkeith Road Edinburgh EH16 4SA, UK; lyon_richard@hotmail.com

Accepted for publication 31 May 2004
Objective: To find out if the response time, distance travelled to scene, and geographical location affect survival from out of hospital cardiac arrest (OHCA).

Methods: Retrospective cohort study over 10 years, 1 December 1991 to 1 August 2001. Outcome and demographic data were obtained for 1956 OHCAs occurring at home, in the Lothians region, from the Heartstart Scotland database. Survival rates to hospital admission and discharge were examined by postcode district.

Results: Certain postcode areas were served by quicker response times and shorter journeys. The survival to admission rate was greater in those areas where the median response time was $<10$ minutes $(13.5 \%$ versus $8.1 \%, \mathrm{p}<0.05)$. There was greater survival to discharge in these areas but not significantly so $(4.1 \%$ versus $3.2 \%, p=0.42)$. Survival to admission was more likely in areas where the median distance travelled was $<$ four miles (13.3\% versus $9.7 \%, p<0.05)$ but not survival to discharge $(4.0 \%$ versus $3.7 \%, p=0.72)$. A close correlation between distance travelled and response time was shown (Pearson $=0.93, p<0.01$ ), likewise between distance travelled and survival to admission (Spearman $=-0.87, p<0.01$ ). Certain areas, particularly those at greater geographical distance from ambulance dispatch points, were found to be associated with significantly lower survival to admission rates.

Conclusions: Survival to admission from OHCA is strongly influenced by response time and distance travelled to the scene. The geographical location of an arrest can potentially influence survival to admission. Measures should be taken to strategically position ambulance dispatch points and to task the nearest geographically available vehicle to attend an OHCA.

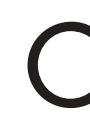
ardiac arrest remains a leading cause of cardiovascular mortality with about 123 events occurring per year per 100000 of the UK population, ${ }^{1}$ with around 50\% caused by acute myocardial infarction. The survival rate for out of hospital cardiac arrest (OHCA) (served by a defibrillator equipped ambulance service) averages only $6.4 \%{ }^{2}$ The Department of Health has drawn up a national service framework for reducing mortality from coronary heart disease $^{1}$ and specifically emphasises the need for a defibrillator to be brought rapidly to all patients with suspected myocardial infarction. The "chain of survival" concept entailing prompt effective cardiopulmonary resuscitation (CPR), rapid defibrillation, and rapid ambulance response time has been shown to reduce OHCA mortality ${ }^{3}$ and is the focus of survival rate improvement.

Despite many advances in resuscitation and emergency cardiac care, it is recognised that the administration of effective CPR and rapid defibrillation remain the key factors that determine outcome. ${ }^{45} \mathrm{CPR}$ training programmes aim to increase the percentage of the general population trained in basic life support, as early CPR increases the survival rate of OHCA patients. ${ }^{6}$

Current ambulance response standards require a crew to reach $75 \%$ of cardiac arrests within eight minutes. The effect of reducing the response time to five minutes increases the rate of survival to discharge to $10 \%-11 \%,{ }^{7}$ a relative increase of $56 \%-71 \%$. High rates of survival have been reported when an OHCA occurs in the presence of an ambulance crew or GP equipped with a defibrillator. ${ }^{6}{ }^{7}$ Ideally, a defibrillator would be available the instant the cardiac arrest occurred as the chances of a successful outcome diminish by about $10 \%$ with every minute that passes. ${ }^{8}$ Although planned for the future, current Scottish Ambulance Service dispatch systems have no means of being able to reliably dispatch the nearest geographically available crew to the scene of an OHCA.

A complementary approach to reduce response times entails equipping other emergency services, such as fire and police, with automated external defibrillators (AEDs). While this can be an effective means of providing rapid defibrillation, some studies have shown that as they are often dispatched from central locations, similar to ambulance crews, they have little influence on OHCA survival. ${ }^{9-11}$ Thus, the specific local organisation of such a system has a large bearing on OHCA outcome.

The purpose of this study is to identify whether response times and survival from OHCA in south east Scotland vary according to geographical location. The study uses postcode areas to subdivide the Lothians region of Scotland, from which the study cohort is derived. Postcode data are used to identify the relations between the timings of cardiac arrest, the distance travelled by ambulance crews to the scene, and outcome. Implications for the ambulance service and for organisation of the dispatch system are discussed.

\section{METHODS \\ Data}

The Heartstart Scotland group keeps detailed records of all out of hospital resuscitation attempts. ${ }^{42}$ Ambulance crews record details of cardiac arrests, resuscitation, and outcome. After each resuscitation attempt, the attending ambulance technician/paramedic(s) complete a standardised data form. This details the patient information, events before arrival of

Abbreviations: OHCA, out of hospital cardiac arrest; CPR, cardiopulmonary resuscitation; $\mathrm{AED}$, automated external defibrillator; $I H D$, ischaemic heart disease 
the ambulance crew, ambulance response time, (time from receipt of the 999 call to arrival on scene-this is recorded automatically by the ambulance service's computer control system) and arrest and defibrillation details. Initial rhythm data upon arrival of the ambulance crew were not recorded. Ambulances are dispatched from one of eight ambulance stations, from either of the two acute receiving hospitals in Edinburgh city (WGH, Western General Hospital, RIE, Royal Infirmary of Edinburgh), or while travelling between dispatch points. Patients are taken to one of four hospitals in the Lothian and Border's region. No significant change in this system occurred during the study period. The final outcome is noted, with data from cases surviving to reach hospital being entered by hospital practitioners at a later date.

Data from the Heartstart Scotland database were extracted for a 10 year period between December 1991 and August 2001 for cardiac arrests attended in Lothian and part of Borders region, specifically those occurring in an $\mathrm{EH}$ postcode district. This area covers a total of $6597 \mathrm{~km}^{2}$, representing a population of 885950 . The proportion of males was $47.7 \%$ and females $52.3 \%$.

All cardiac arrests of presumed cardiac aetiology were included. These included cases where the probable cause of cardiac arrest was stated to be "heart disease" on the Heartstart form. Cases where the probable cause was stated as drowning, drug overdose, asphyxiation, cerebrovascular accident, trauma, suicide, or other cause were excluded. Only cardiac arrests that occurred within the patient's home, where the 999 call was made after the cardiac arrest occurred were selected. The patient's address was used to establish in which postcode district the event occurred. Microsoft Autoroute 2001 (contained in Microsoft Works Suite, 2001) was used. Those records outwith the EH area or where the postcode could not be accurately established were discounted $(\mathrm{n}=27$, total $=1956)$. Ambulance dispatch points were also determined.

\section{Analysis}

Primary analysis

We used $2 \times 2 \chi^{2}$ contingency table analysis to compare survival rates in distinct populations relating to response time, distance travelled to scene, and postcode district in which the event occurred. We used one degree of freedom with a $\mathrm{p}$ value of 0.05 taken to indicate significance. Where a data value was less than 5 Fisher's exact test was applied.

(1) The primary analysis was to establish the relation between survival rate and postcode district. Cardiac events occurring within one postcode district of an ambulance dispatch point were compared with those occurring further away. The main outcome measures were survival to hospital admission and to discharge. Records that had incomplete outcome data were discounted $(\mathrm{n}=97)$.

(2) The second analysis was to establish whether a relation exists between the survival rate from OHCA and the distance from Edinburgh city, where the two acute receiving hospitals are situated. The limit of the city was defined by the Edinburgh city bypass road (A720). OHCA events occurring within this area were compared with those occurring out with this area. Statistical methods as above were applied.

Based on the findings from the primary analyses post hoc analysis was performed.

Post hoc analysis

(3) We examined the relation between survival rate from OHCA and response time. Data were divided into quintiles by response time. Outcome measures were survival to hospital admission and discharge, which were calculated as a proportion of each quintile. The median response time was then plotted against the survival to hospital admission and discharge rates.

(4) The relation between OHCA survival and the distance travelled to the scene was examined in a similar manner, data divided into quintiles according to distance travelled. As the data showed a parametric distribution, a Pearson correlation coefficient was used to examine the relation between distance travelled and response time.

(5) To determine whether a threshold relation existed between response time and survival, temporal data were examined according to individual districts. Where the number of records for an individual district was less than 5 , these records were included into the next geographically closest district. Median response time and median distance travelled were calculated, together with the survival rates. Districts with a median response time greater than 10 minutes were compared with those districts with shorter response times with respect to survival to admission. A similar analysis was used to compare districts with a median response of four miles, or more, with those associated with shorter response distances.

(6) Maps were created using ESRI Arc View Geo Information System. Areas with consistently slow response times and low survival rates were identified and their mortality rates compared with the remaining $\mathrm{EH}$ postcode districts.

(7) The correlation between response time and distance travelled was established by comparing the median distance travelled to the median response time fields of individual postcode districts. As the data showed a parametric distribution a Pearson correlation was used.

\section{RESULTS}

Within the pre-defined region, 1956 patients suffered an OHCA, of suspected cardiac origin, in their own home, during the study period. The male:female ratio was $67.8: 31.9 \%$, with sex not being recorded in three $(0.15 \%)$ of cases. The age of the patient was noted in $1452(74.2 \%)$ of cases, with the average age found to be 69.8 years. A total of 939 (48.0\%) patients were noted to have had previous ischaemic heart disease (IHD). Bystander CPR was performed before the arrival of the ambulance crew in $743(38.0 \%)$ of cases. CPR was most commonly performed by one of the patient's relatives $(n=399,53.7 \%)$.

An EH postcode could be accurately established in 1929 $(98.6 \%)$ cases. The home postcode could not be established in $27(1.4 \%)$ of cases. The final outcome record was available for $1861(95.2 \%)$ cases. In total, $221(11.9 \%)$ patients survived to hospital admission and 72 (3.9\%) survived to hospital discharge.

\section{Primary analysis}

No overall, statistically significant, pattern was seen on analysing the relation between survival rate and postcode district. Patterns analysed included comparing the survival rate in areas within one and two postcode districts from an ambulance dispatch point. Neither was a significant pattern seen in the relation between the survival rate from OHCA and the distance from Edinburgh city. This was analysed by grouping postcode districts into concentric groups radiating away from the city centre. There was a trend toward increased response times and response distances in postcode districts located between ambulance dispatch points (see figs 1 and 2).

\section{Post hoc analysis Response time}

Response time data were available in all of the cases in which an EH postcode was established. Of these, outcome data were 


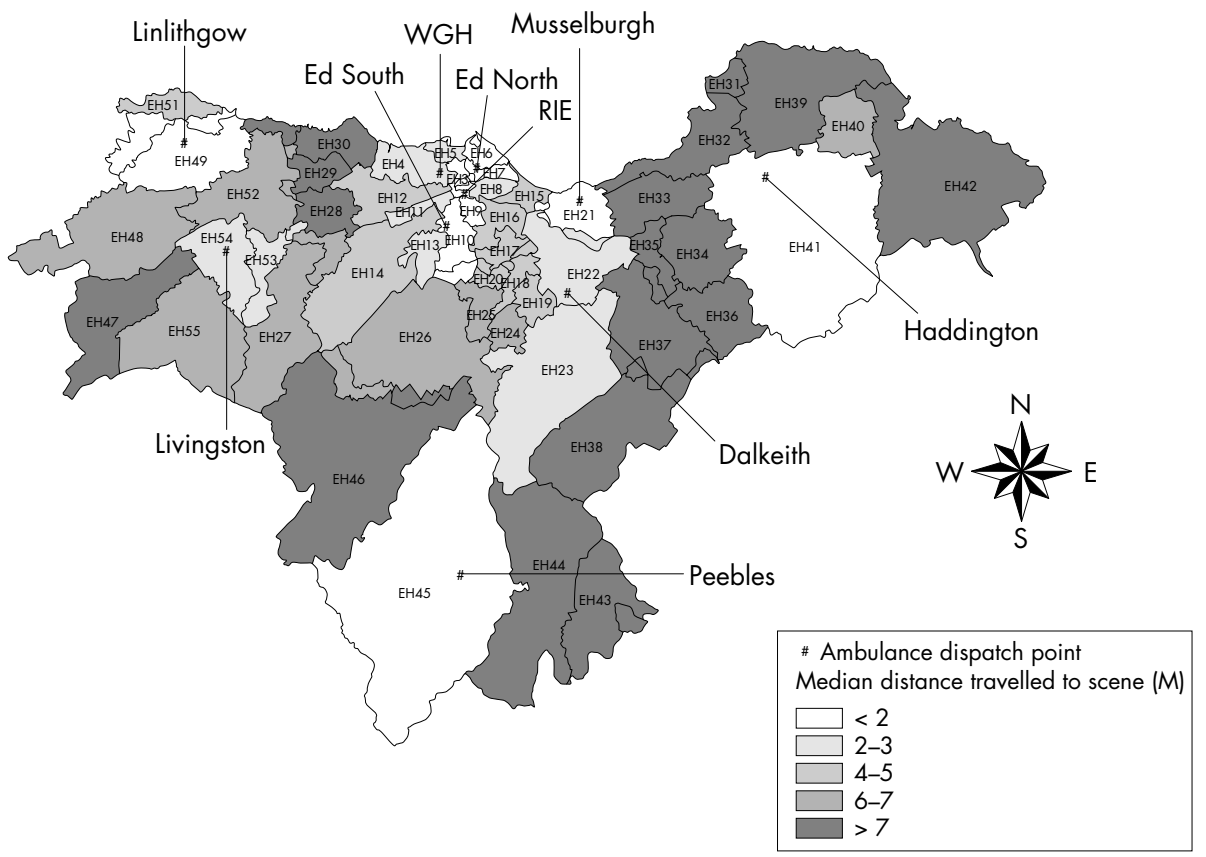

Figure 1 Distance travelled to scene.

available in $1861(95.2 \%)$ cases. Individual response times were used to calculate the median response time of each postcode district. There was a significant relation between ambulance response time and survival from OHCA occurring at home. The mean response time as calculated by postcode district was found to be 10 minutes. This figure was therefore selected as a cut off point. There was a higher survival to admission rate in those postcode districts served by median ambulance response times of less than or equal to 10 minutes, compared with those served by median times greater than 10 minutes ( 13.5 versus $8.1 \%, \chi^{2}=9.11$, $1 \mathrm{df}$, $\mathrm{p}<0.05)$. A higher survival to discharge rate was seen in those districts served by response time of 10 minutes or less, but not significantly so $\left(4.1\right.$ versus $3.2 \%, \chi^{2}=0.8$, $1 \mathrm{df}$, $\mathrm{p}=0.42$ ).

Data on the response distance were available in all of the cases in which an EH postcode was established. Individual response distance data were used to calculate the median distance travelled in each postcode district. No patient survived when the distance travelled exceeded 12 miles. When distance travelled versus proportion of OHCA patients surviving to admission was performed a Spearman correlation of $-0.87(p<0.01)$ was established. A Spearman of 1 would indicate a completely linear relation. The plot of distance travelled versus proportion surviving to hospital discharge gave a Spearman of $-0.16(\mathrm{p}<0.01)$.

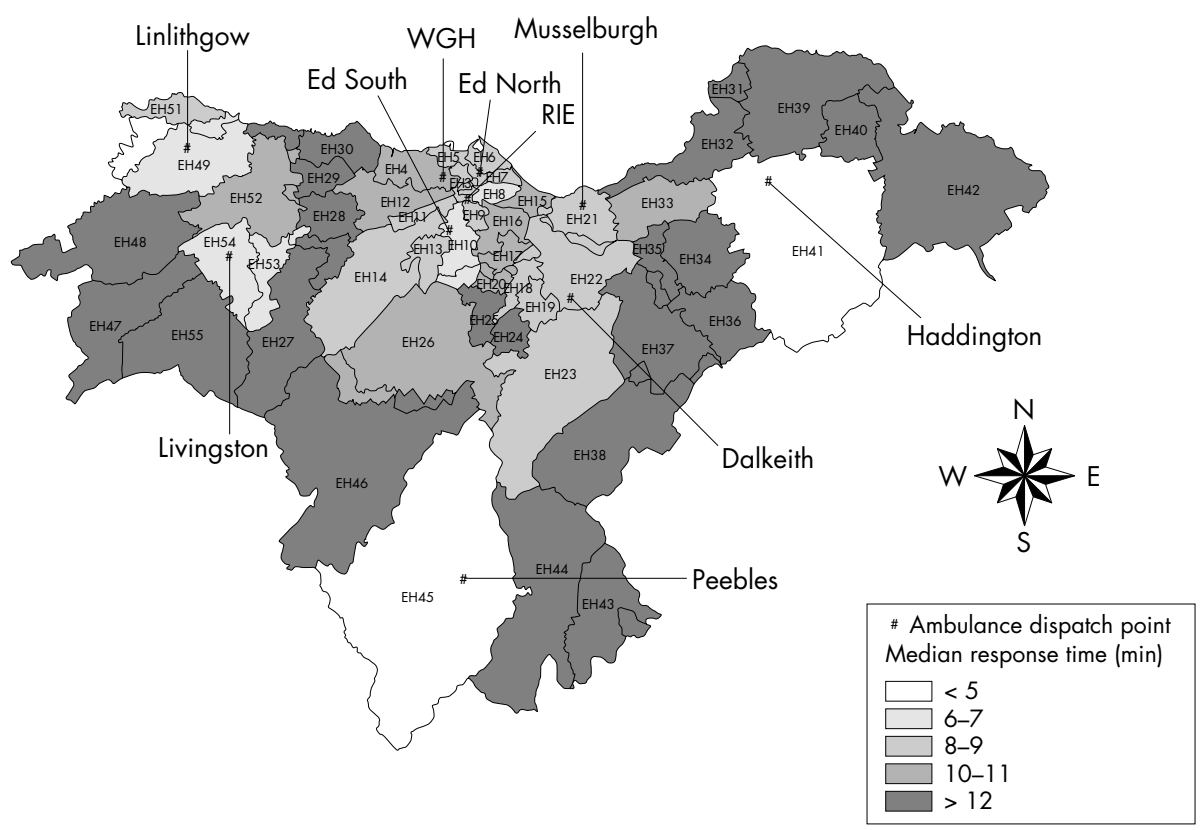

Figure 2 Response time. 
When analysed by postcode district, the median distance travelled was about four miles. This figure was therefore selected as a cut off point. Survival to admission rates were higher in those districts where the median distance travelled by the ambulance was less than four miles ( 13.3 versus $9.7 \%$, $\left.\chi^{2}=4.84, \mathrm{ldf}, \mathrm{p}<0.05\right)$. Survival to discharge rate was higher in those districts with a median distance travelled of less than four miles but no significantly so (4.0 versus $3.7 \%, \chi^{2}=0.12$, ldf).

Distance travelled to scene versus response time Distance travelled data were available in all of the cases in which an EH postcode was established. A significant correlation (Pearson correlation $=0.93, \mathrm{p}<0.01$ ) was established between the distance travelled to the scene of a cardiac event and the response time (fig 3). The median distance travelled was compared with the median response time of each postcode district, giving a total of 44 data points. While this correlation was true in general, certain rural areas (EH45) where the ambulance had a long distance to travel were served by relatively short response times (figs 1 and 2). From the data spread in figure 3 the approximate speed travelled by ambulances when responding to a cardiac arrest call is $35 \mathrm{mph}$. Also to be noted is that when distance $=0$ there is still a median response time of about five minutes.

\section{Postcode regions with lower survival rates}

A number of geographical regions (EH 32-37, EH 31, 39, 40, 42 , EH $47,48,52,55$ ) were shown to have significantly lower survival to admission rates when compared with the rest of the Lothian and Borders region (see fig 4). No significant difference was seen in survival to hospital discharge. These regions did not appear to have a higher incidence of IHD, nor did rates of bystander CPR vary significantly when compared with the rest of the study region. These regions are geographically at an increased distance from ambulance dispatch points (see figs 1 and 2).

\section{DISCUSSION}

This study shows that initial survival from OHCA is influenced by ambulance response time and distance, with

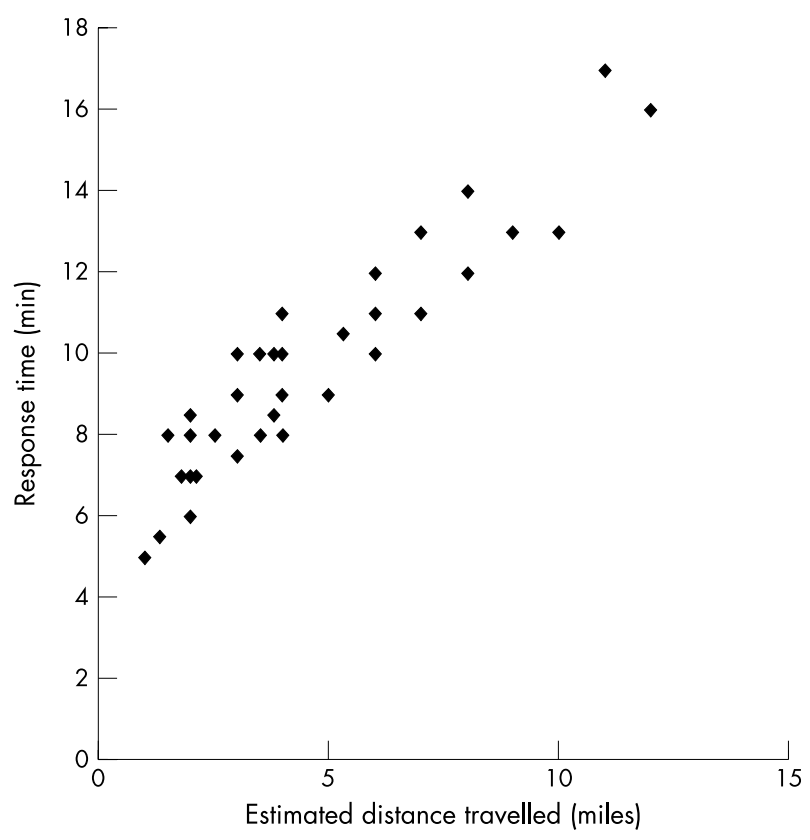

Figure 3 Ambulance response time compared with distance travelled to scene by ambulance when responding to OHCA calls.
12 miles being a cut off point for survival. This study specifically examined OHCA events occurring at home; in which cardiac arrest is less likely to be witnessed and bystander CPR less likely to be administered. Even if bystander CPR is administered, our results show a relative most commonly performs it. It has been suggested, that when a relative of the victim performs CPR, there is no increased chance of survival. ${ }^{5}$ In the UK, $74 \%$ of all cardiac arrests occur outside hospital and $75 \%$ of these occur at home. $^{13}$

The finding of a strong correlation between distance travelled and survival to admission suggests that ambulance dispatch points are key to survival. Areas in between ambulance dispatch points are served by slower response times and associated with greater response distances (figs 1 and 2), with lower initial survival rates than other postcode regions. An effective system of strategically stationing ambulances, or other first responders, together with a geographical tracking system to dispatch the nearest available vehicle, could potentially increase OHCA survival rates. The results show that no patient survived when the distance travelled to scene was greater than 12 miles suggesting an ambulance would have to be within a 12 mile radius of the OHCA scene if there is to be a chance of survival.

The finding that when the distance travelled was 0 the response time was five minutes is interesting. In theory, this figure represents the time taken from initial receipt of the 999 call to the ambulance crew receiving the call details. It could also result from ambulance crews selecting "0 miles" when in reality they travelled a small distance. The theoretical delay occurs as a result of the ambulance operator taking the call, having to transfer the details to a dispatcher who relays the information to the ambulance crew. If not already, the crew have to get to the ambulance once the call has been received. This figure seems larger than expected and warrants further research as it has implications for the dispatch system.

A potential strategy to improve outcome is public access defibrillation. This entails placing AEDs in public places and having designated, trained lay people assigned to attend the cardiac arrest. While studies suggest these AEDs do improve overall survival, ${ }^{14}$ the small number of isolated OHCAs that occur in any given location means that the logistics of such a system are not straightforward..$^{15}$ AEDs in public places are unlikely to play a part in the intervention of cardiac arrests occurring within the patient's home. Community based first responder programmes, based on a Neighbourhood Watch scheme are an alternative strategy. ${ }^{16}$ Although this is yet to be implemented in the UK, it may it be possible to create such schemes and place public access AEDs in areas served by consistently slower ambulance response times. The use of a postcode based system to examine OHCA data has proved to be an effective way of identifying certain individual postcode areas with consistently slow response times and lower than average survival rates. These areas could be targeted for the implementation of first responder AED programmes. ${ }^{9-11} 14$

The finding that the two primary hypotheses were not supported may reflect the limited statistical power of the study to show differences in survival to discharge. One reason for failing to establish a significant geographical pattern with respect to OHCA survival may be that ambulances are often dispatched en route from locations other than their home station. This would affect the response time and distance travelled to scene data with respect to specific postcode districts and may potentially compensate for the scarcity of dispatch stations. Ambulance crews are required to provide an estimate of the distance they travelled to the scene of an OHCA. The increase in survival rate at the five mile point could therefore be attributable to digit preference. 


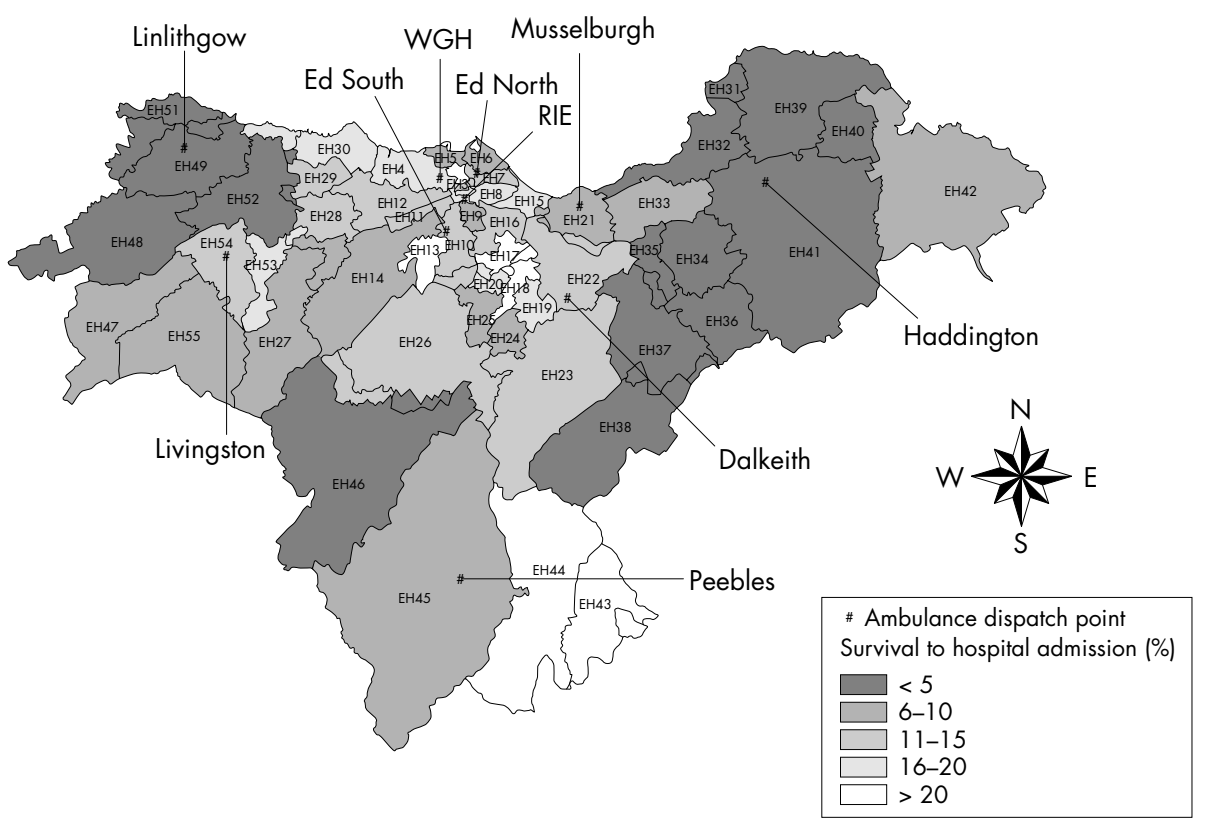

Figure 4 Survival to hospital admission.

It was assumed that most cardiac arrests occurring in the $\mathrm{EH}$ area over the 10 year time period were accounted for. As complete data were available in $95 \%$ of the reported OHCAs it was assumed most Heartstart questionnaires had been completed diligently.

None of the variables examined significantly affected survival to hospital discharge; which is the most important outcome measure. As the percentage of patients surviving to hospital discharge was so low (3.9\%), the study may not have been sufficiently powerful to detect factors influencing survival to discharge rate. Some areas had only five cases (for example, EH 43,44) with a single survivor resulting in a high survival to discharge rate. Potential confounding factors include the incidence of IHD and the rate of bystander CPR. Bystander CPR given by someone unrelated to the patient was given in only $17 \%$ of cases and there was no significant variation across the study region. The incidence of IHD did vary in certain regions. While these confounding factors did not figure in the areas we identified as having lower survival to admission rates, they may be confounding the primary hypothesis. Other variables could also be discounting the effect of response time and distance travelled to the scene. A potential weakness of the study is that information on initial cardiac rhythm on arrival of the ambulance crew was not available. Postmortem examination findings were also not examined. The low overall survival could result from the fact that cardiac arrests occurring out with the home carry a higher chance of bystander CPR and hence survival. ${ }^{12}$

The Lothian and Borders region of Scotland is a good example of several urban areas, where most of the ambulance dispatch points are located, with suburban and rural areas located in between. Many regions in the UK and Europe are likely to have a similar ambulance dispatch system and thus show a similar pattern of results.

\section{CONCLUSION}

It has been shown that initial survival from OHCA is influenced by geographical location in certain discrete geographical areas. Further research is warranted into the time taken to dispatch an ambulance to an OHCA call. It is possible to identify areas that show consistently slow response times. Cutting response times, establishing first responder programmes in strategic geographical areas, and using geographical systems to task the nearest available responder to the scene of an OHCA could potentially save lives.

\section{ACKNOWLEDGEMENTS}

The authors wish to thank the following people for their support: Dr Colin Robertson for assistance with the manuscript. The technicians and paramedics of the Scottish Ambulance Service for their diligence in treating the patients under their care and completing the Heartstart questionnaires. Miss Robin Rice, University of Edinburgh Data Library, for her assistance in producing the postcode maps.

\section{Authors' affiliations}

R M Lyon, N R Grubb, Department of Cardiology, Royal Infirmary of Edinburgh, UK

S M Cobbe, J M Bradley, Department of Medical Cardiology, Glasgow Royal Infirmary, UK

Funding: none.

Conflicts of interest: none declared.

\section{REFERENCES}

1 Norris RM, on behalf of the UK Heart Attack Study investigators. Sudden cardiac death and acute myocardial infarction in three British health districts: the UK heart attack study. London: British Heart Foundation, 1999.

2 Nichol G, Stiell IG Laupacis A, et al. A cumulative meta-analysis of the effectiveness of defibrillator-capable emergency medical services for victims of out-of-hospital cardiac arrest. Ann Emerg Med 1999;34:517-25.

3 Eisenberg MS, Coppas MK, Hallstrom AP, et al. Treatment of out-of-hospital cardiac arrest with rapid defibrillation by emergency medical technicians. N Engl J Med 1980;302:1379-83.

4 Cobbe SM, Redmond MJ, Watson JM, et al. "Heartstart Scotland" - initial experience of a national scheme for out of hospital defibrillation. BMJ 1991;302:1517-20.

5 Grubb NR, Elton RA, Fox KAA. In-hospital mortality after out-of-hospital cardiac arrest. Lancet 1995;436:417-21.

6 Rea TD, Eisenberg MS, Culley LL, et al. Dispatcher-assisted cardio-pulmonary resuscitation and survival in cardiac arrest. Circulation 2001;104:2513-16.

7 Pell JP, Sirel JM, Marsden AK, et al. Effect of reducing ambulance response times on deaths from out of hospital cardiac arrest: cohort study. BMJ 2001;322:1385-8.

8 Larsen MP, Eisenberg MS, Cummins RO, et al. Predicting survival from out of hospital cardiac arrest: a graphic model. Ann Emerg Med 1993;22:1652-8.

9 Smith KL, Peeters A, McNeil JJ. Results from the first 12 months of a fire firstresponder program in Australia. Resuscitation 2001;49:143-50.

10 Forrer CS, Swor RA, Jackson RE, et al. Estimated cost effectiveness of a police automated external defibrillator program in a suburban community: 7 years experience. Resuscitation 2002;52:23-9. 
11 Groh WJ, Newman MM, Beal PE, et al. Limited response to cardiac arrest by police equipped with automated external defibrillators: lack of survival benefit in suburban and rural Indiana: the Police as Responder Automated Defibrillation Evaluation (PARADE). Acad Emerg Med $2001 ; 8: 324-30$.

12 Sedgwick ML, Dalziel K, Watson J, et al. Performance of an established system of first responder out-of-hospital defibrillation. The results of the second year of the Heartstart Scotland project in the "Utstein Style". Resuscitation 1993;26:75-88.
13 Norris RM. Fatality outside hospital from acute coronary events in three British health districts, 1994-5. United Kingdom Heart Attack Study Collaborative Group. BMJ 1998;316:1065-70

14 Wollard M. Public access defibrillation: a shocking idea? J Public Health Med 2001:23:98-102.

15 Gratton M, Lindholm DJ, Campbell JP. Public-access defibrillation: where do we place the AEDs? Prehospital Emergency Care 1999;3:303-5.

16 Zipes DP. President's page: the neighborhood watch programme: Save A Victim Everywhere (SAVE). J Am Coll Cardiol 2001;37:2004-5. 